\title{
Numerical and Experimental Analysis of the Hydrodynamic Performance of a Three-Dimensional Finite-Length Rotating Cylinder
}

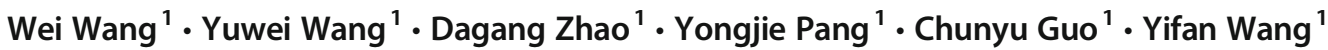

Received: 6 November 2019 / Accepted: 20 April 2020 / Published online: 8 October 2020

(C) The Author(s) 2020

\begin{abstract}
The hydrodynamic performance of a three-dimensional finite-length rotating cylinder is studied by means of a physical tank and numerical simulation. First, according to the identified influencing factors, a hydrodynamic performance test of the rotating cylinder was carried out in a circulating water tank. In order to explore the changing law of hydrodynamic performance with these factors, a particle image velocimetry device was used to monitor the flow field. Subsequently, a computational field dynamics numerical simulation method was used to simulate the flow field, followed by an analysis of the effects of speed ratio, Reynolds number, and aspect ratio on the flow field. The results show that the lift coefficient and drag coefficient of the cylinder increase first and then decrease with the increase of the rotational speed ratio. The trend of numerical simulation and experimental results is similar.
\end{abstract}

Keywords Rotating cylinder $\cdot$ Magnus $\cdot$ Rotational speed ratio $\cdot$ Aspect ratio $\cdot$ Computational field dynamics $\cdot$ Model test

\section{Introduction}

The hydrodynamic performance of cylinders is a classic and complex problem in fluid mechanics research, and has wide applications in scientific research and engineering (e.g., aerospace research, water conservation, and marine engineering). Due to the physical structure of a cylinder, its flow field and force characteristics are affected by several factors, such as the Reynolds number, surface roughness, cylinder size, and turbulence intensity. In many engineering situations (Sedaghat et al. 2014; Liang et al. 2016; Ommani et al. 2016), the cylinders in a structure must be rotated at a certain rate; hence,

\section{Article Highlights}

- In order to meet the practical application in engineering, it is necessary to study the hydrodynamic performance of rotating cylinder under different conditions.

- Both physical experiment and PIV test of the rotating cylinder were carried out in the circulating water channel.

- The hydrodynamic performance of a rotating cylinder was simulated under three parameters and compared with the experimental results.

Dagang Zhao

zhaodagang@hrbeu.edu.cn

1 College of Shipbuilding Engineering, Harbin Engineering University, Harbin 150001, China studying the hydrodynamic performance of a rotating cylinder is a critical endeavor.

The problem of trajectory deflection caused by rotating cylinders was first discovered and discussed in the seventeenth century. In 1852, the German scientist, Magnus, described this phenomenon in detail, explaining the deflection of a rotating object. Hence, this phenomenon, namely, the Magnus effect, is named after him (Fleming and Probert 1984). Meanwhile, the difference in flow around a rotating cylinder and a smooth cylinder is manifested in two aspects: (1) the cylinder has rotational speed, (2) and the cylinder surface is rough (when considering fluid viscosity). If the cylinder surface is smooth, its rotation will not have any effect on the flow field (Du 2016). According to the classification of research methods, related research can be summarized from three aspects: theoretical research, physical model tests, and numerical simulation.

In the twentieth century, a few studies on theoretical calculations of rotating cylinders were performed (Guo 1988; Cheng et al. 1990; Liu and Zhuang 1994; Xu et al. 1995), and research into the applications of these cylinders was also initiated during this period (Zheng and Xu 1993. Rotating cylinder rudders (Xu and Liu 1988; Zhang and Xu 1991; Xu and Sun 1992), which were developed based on the Magnus effect, were widely used in the 1990s. Tu et al. (2008) studied 
the flow around a circular cylinder by using particle image velocimetry (PIV). Karabelas (2010) computed the uniform flow passing a rotating cylinder at $R e=140000$ based on large-eddy simulation. To simulate the flow around a rectangular cylinder with different aspect ratios, Islam et al. (2012) utilized the incompressible lattice Boltzmann method for a two-dimensional (2D) flow. van Rees et al. (2015) used a $2 \mathrm{D}$ viscous flow numerical algorithm to study a pair of cylinders rotating in opposite directions. Meanwhile, Yuan (2016) applied the Magnus effect on wind turbines and, based on such an effect, proposed methods for improving the characteristics of wind turbines with a low lift-to-drag ratio. Zheng et al. (2017) studied the flow passing a spinning circular cylinder at different Reynolds numbers, using the Navier-Stokes equations and the $\gamma-\operatorname{Re} \theta$ transition model, coupled with the SST k $-\omega$ turbulence model based on the finite volume method. Siddiqui (2016) formulated the non-steady flow due to a uniformly accelerated and rotating circular cylinder from rest in a stationary, viscous, incompressible, and micropolar fluid. Wong et al. (2017) investigated a cylinder that rotated as a result of flowing motion, while $\mathrm{Wu}$ et al. (2018) performed a pneumatic numerical simulation on particularly low-speed fin-stabilized rotating projectiles. Finally, Chen et al. (2018) proposed that the Magnus effect can be simulated using steady-state methods by employing numerical simulation methods to calculate the effect of projectile rotations.

In the current study, the commercial CFD software Star-CCM+, which was developed by CD-Adapco, was used to simulate a rotating cylinder under different influencing factors, and the hydrodynamic performance of a three-dimensional (3D) finite-length rotating cylinder was studied. Thereafter, we performed a corresponding model test in a circulation tank to verify the simulation results, followed by a PIV flow field measurement test of the flow around the rotating cylinder. The results of this study will contribute to the development of a Magnus anti-rolling device and help promote further research on ship energy-saving rotor sails.

\section{Governing Equations of Fluid}

The mass conservation equation of fluid mechanics can be written as:

$\frac{\partial u_{x}}{\partial x}+\frac{\partial u_{y}}{\partial y}+\frac{\partial u_{z}}{\partial z}=0$

where $u$ represents the component of fluid velocity on the $x, y$, and $z$ axes.

The momentum conservation equation can be written as:
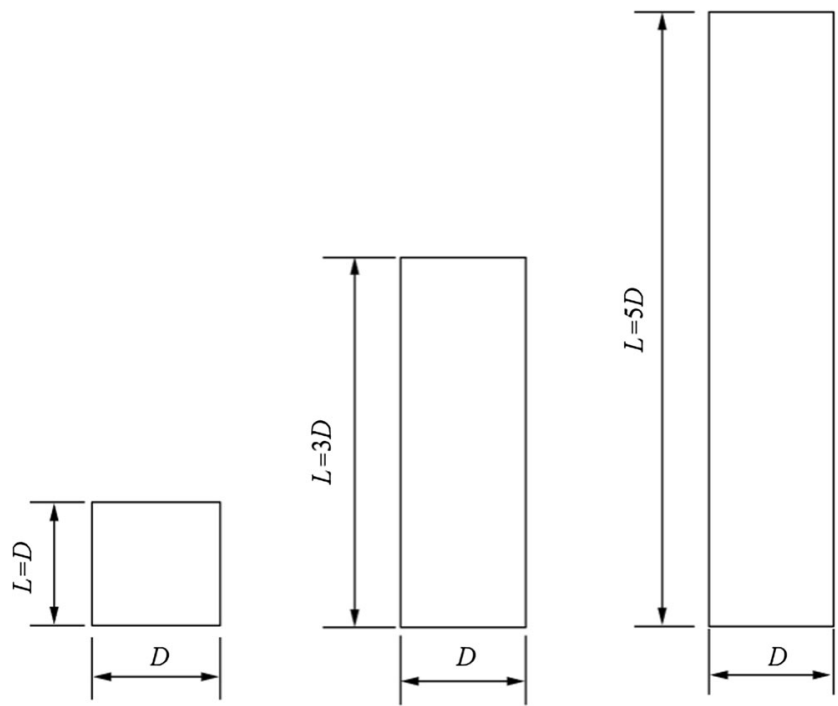

Figure 1 Geometric model size of the cylinders. $L$ length, $D$ diameter

$$
\begin{aligned}
& \frac{\partial u_{x}}{\partial t}+\frac{\partial\left(u_{x} u_{x}\right)}{\partial x}+\frac{\partial\left(u_{x} u_{y}\right)}{\partial y}+\frac{\partial\left(u_{x} u_{z}\right)}{\partial z}=v\left(\frac{\partial^{2} u_{x}}{\partial x^{2}}+\frac{\partial^{2} u_{x}}{\partial y^{2}}+\frac{\partial^{2} u_{x}}{\partial z^{2}}\right)-\frac{1}{\rho} \frac{\partial p}{\partial x} \\
& \frac{\partial u_{y}}{\partial t}+\frac{\partial\left(u_{y} u_{x}\right)}{\partial x}+\frac{\partial\left(u_{y} u_{y}\right)}{\partial y}+\frac{\partial\left(u_{y} u_{z}\right)}{\partial z}=v\left(\frac{\partial^{2} u_{y}}{\partial x^{2}}+\frac{\partial^{2} u_{y}}{\partial y^{2}}+\frac{\partial^{2} u_{z}}{\partial z^{2}}\right)-\frac{1}{\rho} \frac{\partial p}{\partial y}-g \\
& \frac{\partial u_{z}}{\partial t}+\frac{\partial\left(u_{z} u_{x}\right)}{\partial x}+\frac{\partial\left(u_{z} u_{y}\right)}{\partial y}+\frac{\partial\left(u_{z} u_{z}\right)}{\partial z}=v\left(\frac{\partial^{2} u_{z}}{\partial x^{2}}+\frac{\partial^{2} u_{z}}{\partial y^{2}}+\frac{\partial^{2} u_{z}}{\partial z^{2}}\right)-\frac{1}{\rho} \frac{\partial p}{\partial z}
\end{aligned}
$$

where $p$ and $v$ represent the pressure and the kinematic viscosity of a fluid, respectively.

\section{Physical Modeling and Grid Generation}

The aspect ratio $\beta(L / D)$ is a key factor in understanding finitelength rotating cylinders. In terms of the design for working conditions, existing fundamentals must be combined to emulate the real-life scenarios. Therefore, meticulous consideration has been applied to the aspect ratio. Given that rotating cylinders are usually installed at the bottom of the vessel, the aspect ratio of the device should be low to avoid a negative impact on the vessel's navigation, especially in shallow waters with underlying rocks. Using the parameters of the Maglift series of stabilizer fins by Quantum (USA), the largest aspect ratio in this study was set as $L / D=5(D=0.10 \mathrm{~m})$, with the remaining as $L / D=1,3$, and 5 , as illustrated in Figure 1.

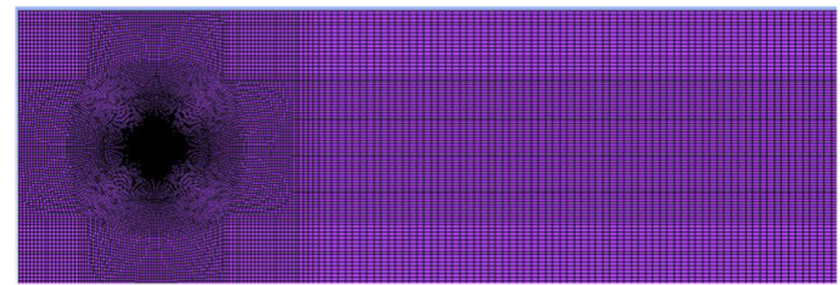

Figure 2 Mesh of three-dimensional finite-length rotating cylinder calculation model 


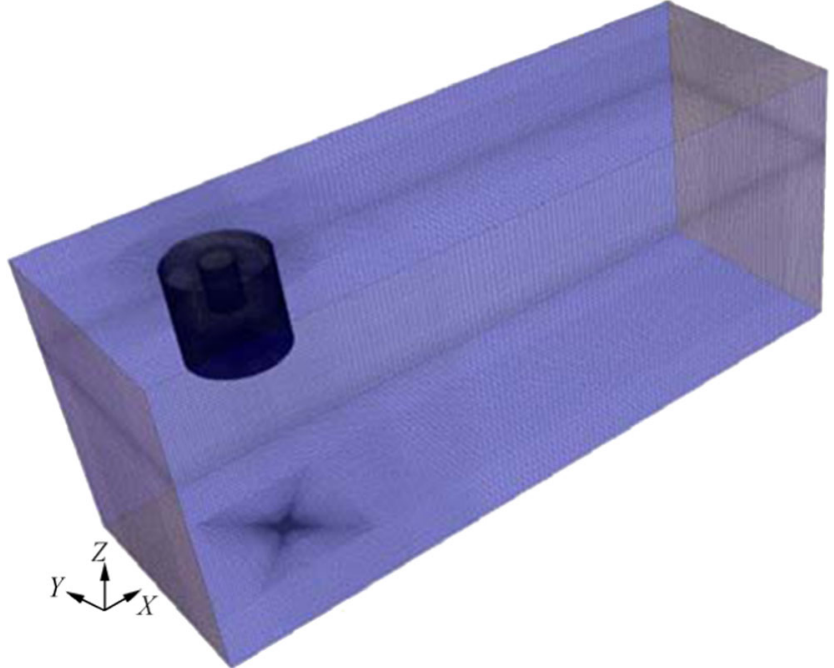

Figure 3 3D model grid diagram

The calculation model for the 3D finite-length rotating cylinder is illustrated in Figure 2. The left side represents the speed inlet, the right side is the pressure outlet, the cylinder surface is set to the wall, and the rest of the domain is the symmetrical boundary. Here, we used a sliding mesh to realize the rotation of the cylinder. The coordinate system in this study was defined as the center of the upper surface of the cylinder as the origin. The positive direction of the $x$ axis was consistent with the incoming flow direction, and the $y$ axis was perpendicular to it. Moreover, the $z$ axis was consistent with the horizontal direction of the cylinder wherein there was drag along the flow direction and lift in the vertical direction. On the basis of a relevant literature review and a comparative analysis of multiple scale computing domains, the computing domain dimension of the 3D flow field was set as $24 \times 8 \times$ $10 D$. We set the left side as the velocity inlet, the right side as the pressure outlet, the cylinder surface as the wall surface,

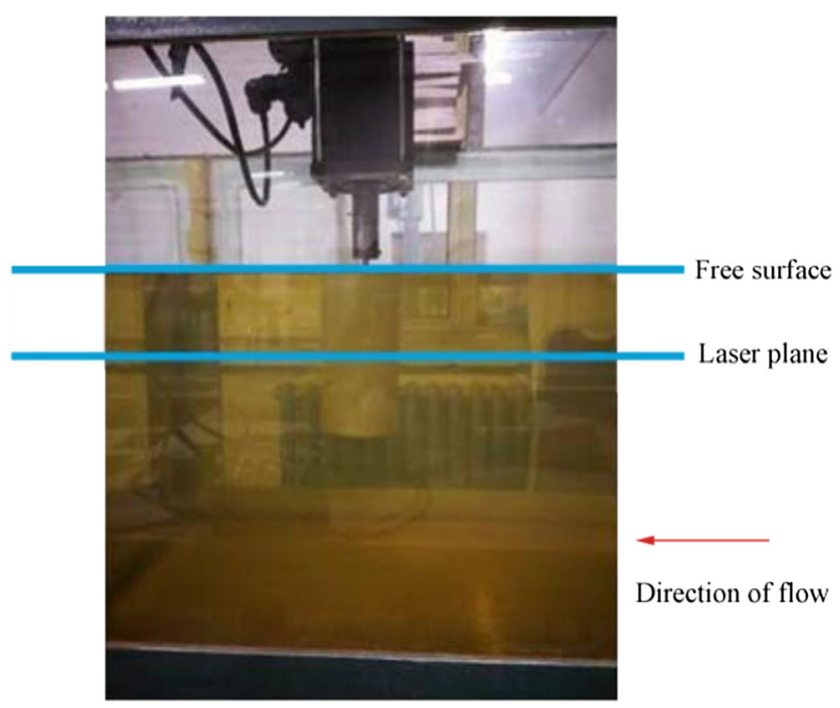

Figure 4 Measurement device and the rest of the domain as the symmetrical boundary. The distance between the velocity inlet and the center of the cylinder was $4 D$, whereas that between the center of the cylinder and pressure outlet was $20 D$. The two sides of the cylinder were symmetrical to the center of the cylinder and were both denoted as $4 D$. The grid model of $L / D=3$ is as shown in Figure 3; as can be seen, the interface wrapped around the

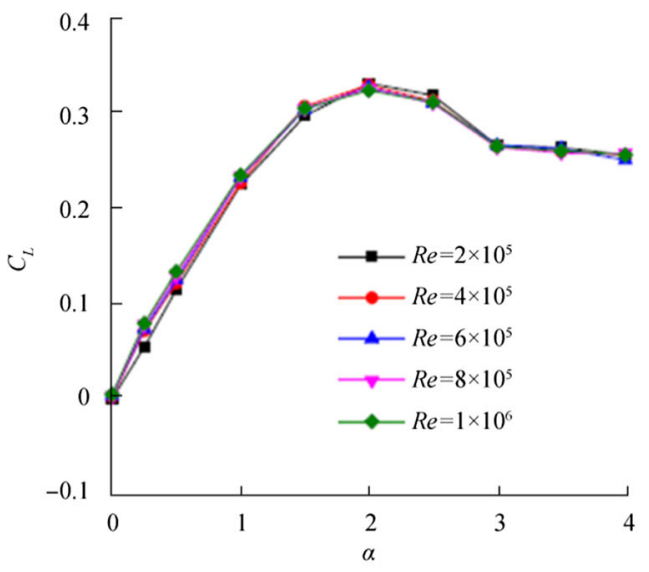

(a) $C_{L}$

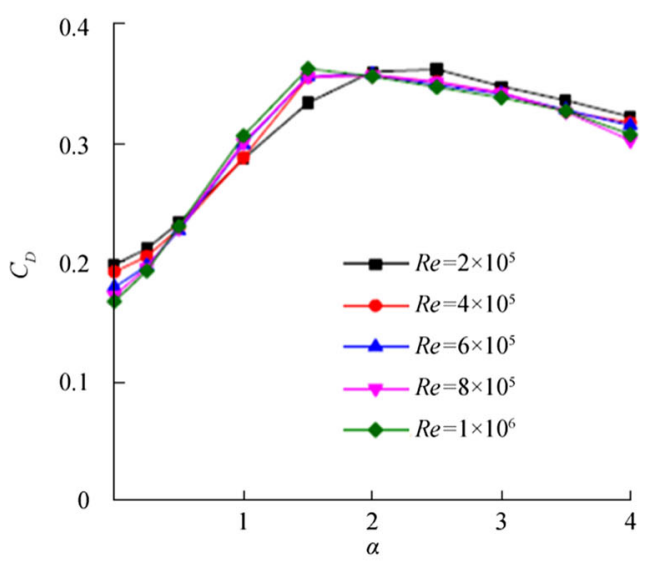

(b) $C_{D}$

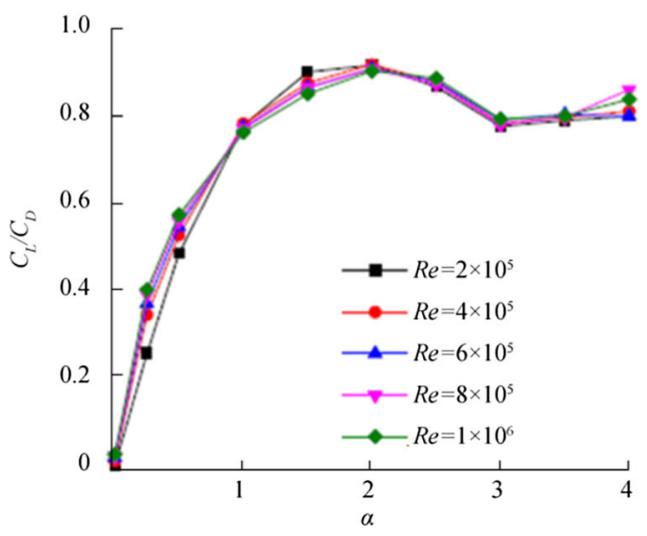

(c) $C_{L} / C_{D}$

Figure 5 Relationships among lift coefficient, drag coefficient, and liftto-drag ratio with speed ratios under different Reynolds numbers 
cylinder at a certain distance throughout. The numerical simulation of the 3D finite-length rotating cylinder was carried out using detached-eddy simulation. The ICEM CFD software was used to generate the grids for the model. Several zones of increased density were considered for the grids. The density decreased from the region around the wall of the cylinder toward the outside, both in the span-wise and the chord-wise directions. We set the cylinder to rotate clockwise around the $z$ axis. Water was used as the fluid medium, and its temperature was set at $20^{\circ} \mathrm{C}$. Moreover, the density was $998.16 \mathrm{~kg} / \mathrm{m}^{2}$, the kinematic viscosity coefficient was $1.0037 \times 10^{6} \mathrm{~m}^{2} / \mathrm{s}$, and the Reynolds number was $R e=10^{6}$, and $U=10.037 \mathrm{~m} / \mathrm{s}(R e=$

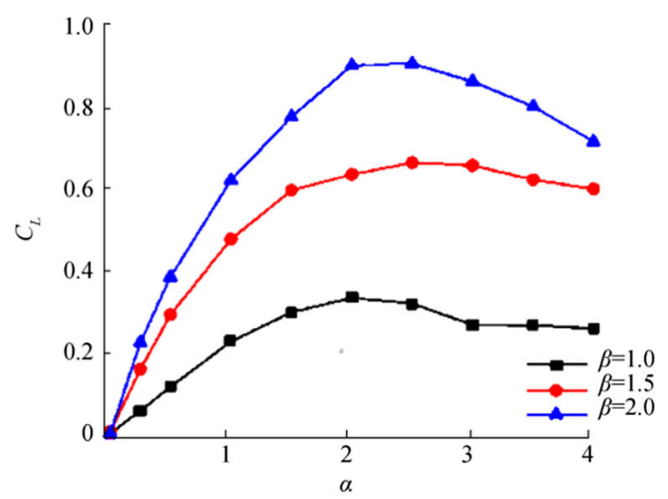

(a) $C_{L}$

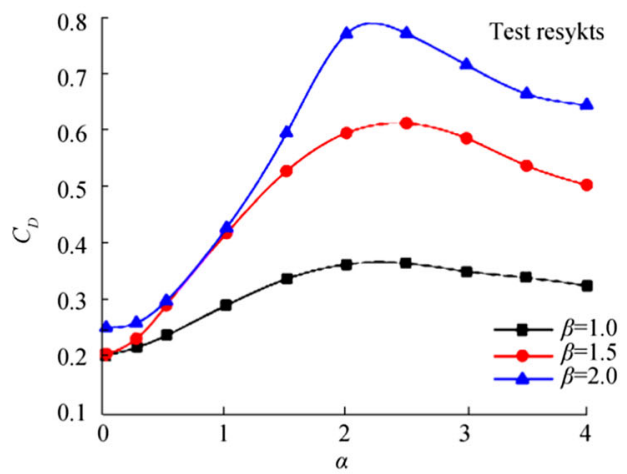

(b) $C_{D}$

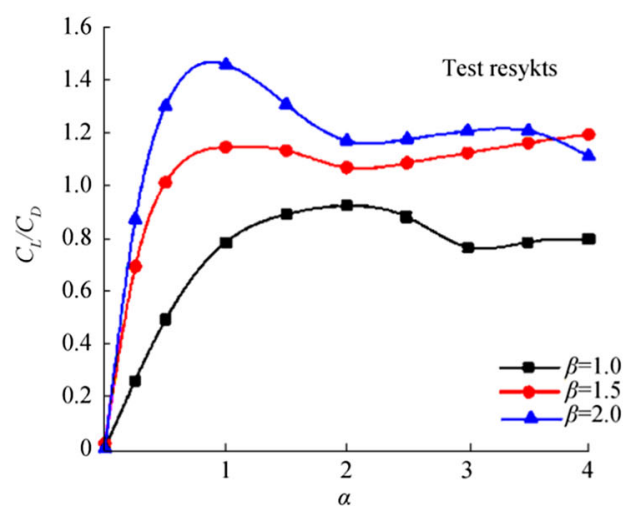

(c) $C_{L} / C_{D}$

Figure 6 Lifting coefficient, drag coefficient, and lift-to-drag ratio with different aspect ratios
$D U / \nu$, where $U$ and $v$ represented the velocity and viscosity of the fluid, respectively). Taking the working condition of the Reynolds number, $R e=2 \times 10^{5}$, and speed ratio, 1 , as examples, the grid independence of the five groups of fluid calculation domains with grid numbers ranging from $0.97 \times 10^{6}$ to $3.58 \times 10^{6}$ were verified. Considering the calculation results and computer resources, the grid number of $2.53 \times 10^{6}$ was selected as the grid number to be simulated in the current study.

\section{Physics Experiment of Rotating Cylinder}

\subsection{Rotating Cylinder Test Model}

The test site was a circulating water channel located in Harbin Engineering University. The velocity was measured using a current meter. In the test device, the cylinder was installed on the motor, which provided the rotation speed. The motor was mounted on a three-component force measuring balance,

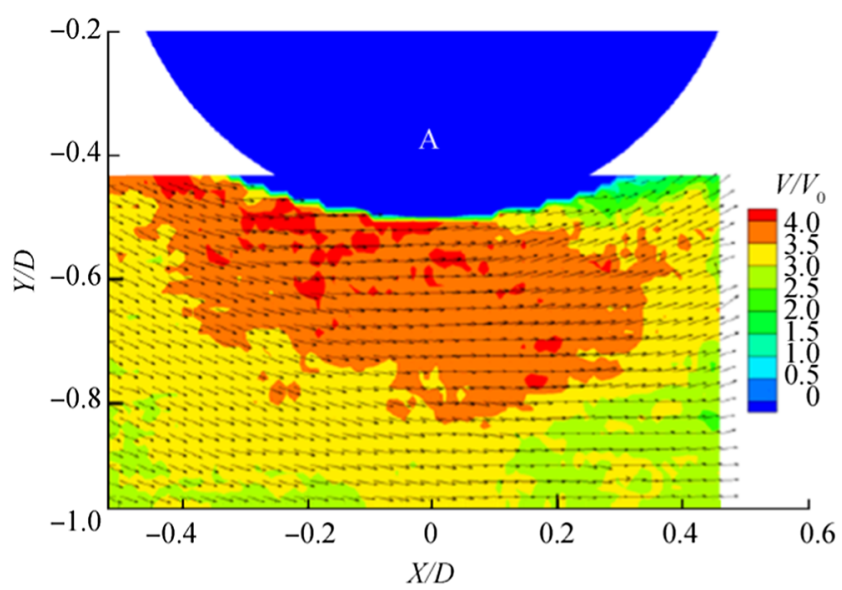

(a) Area $\mathrm{A}$

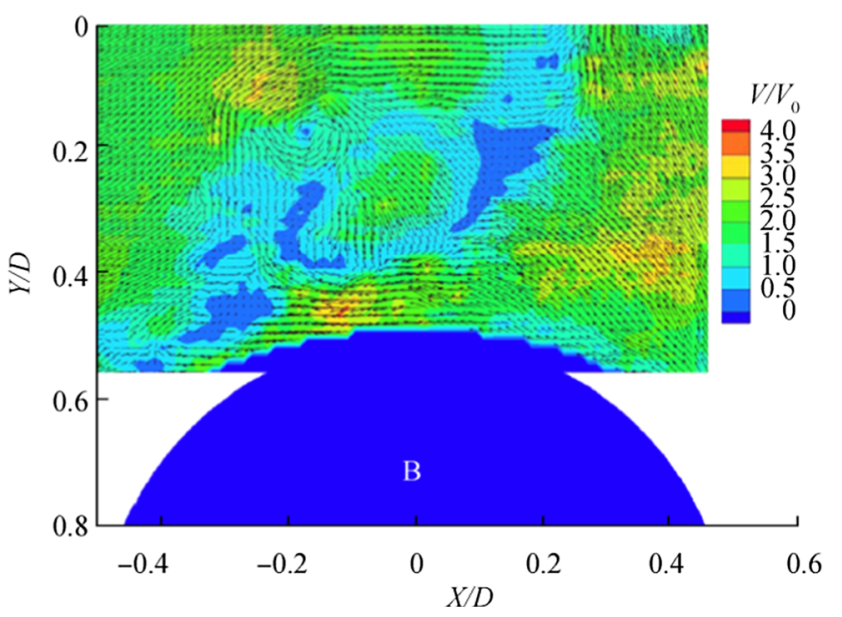

(b) Area B

Figure 7 The flow field diagram measured by PIV 
which measured the resistance and lift of the cylinder. The force balance was fixed above the circulation tank. During the test, a plate was installed on the liquid surface flush with the cylinder in order to eliminate any horizontal disturbance. During the test, we started the channel and motor to ensure that the water flow attained the predetermined speed. Subsequently, the cylinder was started in order to attain its predetermined speed. The force received by the cylinder was measured through the force balance above the cylinder. In addition, the flow field around the cylinder was measured using the PIV device, as shown in Figure 4. The laser horizontally irradiated the cylinder at its middle position with a film light of $1 \mathrm{~mm}$. The tracer particles emitted scattered light, and the camera captured photos from the fixed position at the bottom of the tank.

\subsection{Test Results}

In the experiment, the variable Reynolds number and rotational speed ratio $\alpha(\alpha=\omega D / 2 U$, where $\omega$ is the angular velocity of the cylinder rotation) of model $1(\beta=1)$ were studied. The variable length-diameter ratio was studied at a Reynolds number of $R e=2 \times 10^{5}$. The results are shown in Figure 5.

Here, $C_{L}$ is the lift coefficient $\left(C_{L}=2 F_{L} / \rho U^{2} L D\right.$, where $F_{L}$ is the lift of the cylinder), and $C_{D}$ is the drag coefficient $\left(C_{D}=\right.$ $2 F_{D} / \rho U^{2} L D$, where $F_{D}$ is the drag of the cylinder). The lift-todrag ratio $C_{L} / C_{D}$ is an important index reflecting the anti- rolling effect, i.e., the larger the lift-drag ratio, the better the anti-rolling effect.

With the increase of the rotational speed ratio, the lift coefficient increased sharply in the range of $0-1.5$, but when the rotational speed ratio exceeded 1.5 , the lift coefficient grew slowly or even slightly decreased, and was basically not affected by the Reynolds number. As can be seen from the resistance curve, when the rotational speed ratio was in the range of $0-0.5$, the resistance coefficient decreased with an increase in the Reynolds number; however, it increased with the further increase of the rotational speed ratio. Similarly, the resistance coefficient also increased with an increase in the Reynolds number. Considering the cylinder rotational speed, the flow was actually in the transition zone; thus, the resistance coefficient started to rise. From the lift-drag ratio curve, the liftdrag ratio first increased and then decreased with the increase of the rotational speed ratio, thereby reaching its maximum within the range of 1.5-2.5, indicating that it was not affected by the Reynolds number.

Figure 6 shows the respective lift coefficient, drag coefficient, and lift-to-drag ratio curves of the rotating cylinder model test with different length-diameter ratios under the Reynolds number, $R e=2 \times 10^{5}$. As can be seen, both the lift and drag coefficients increased with an increase in the lengthdiameter ratio. However, the rotational speed ratio corresponding to the maximum lift-to-drag ratio of models with different length-diameter ratios was different. With an
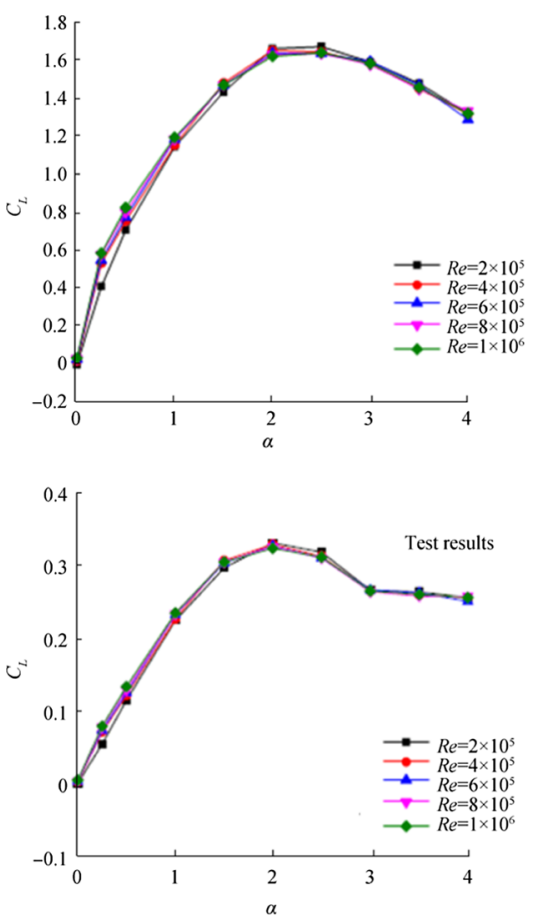

(a) $C_{L}$
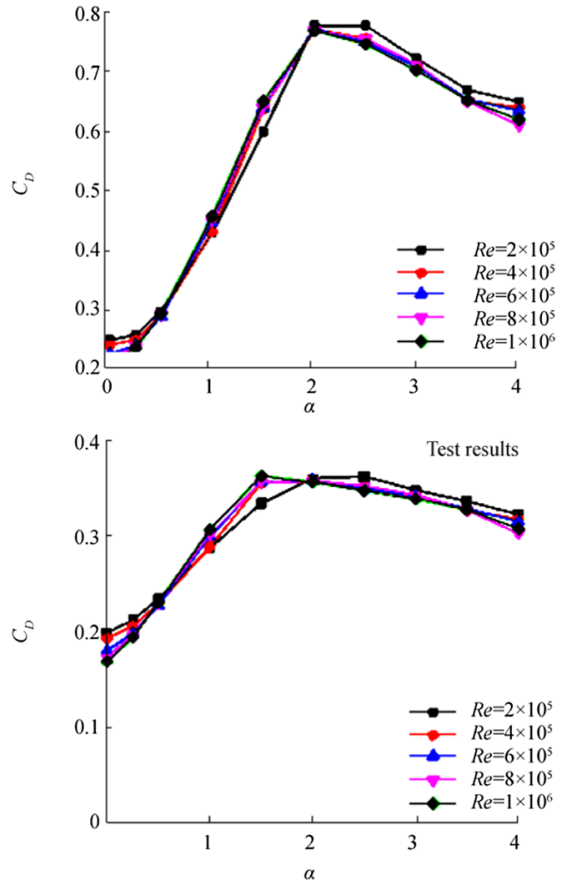

(b) $C_{D}$
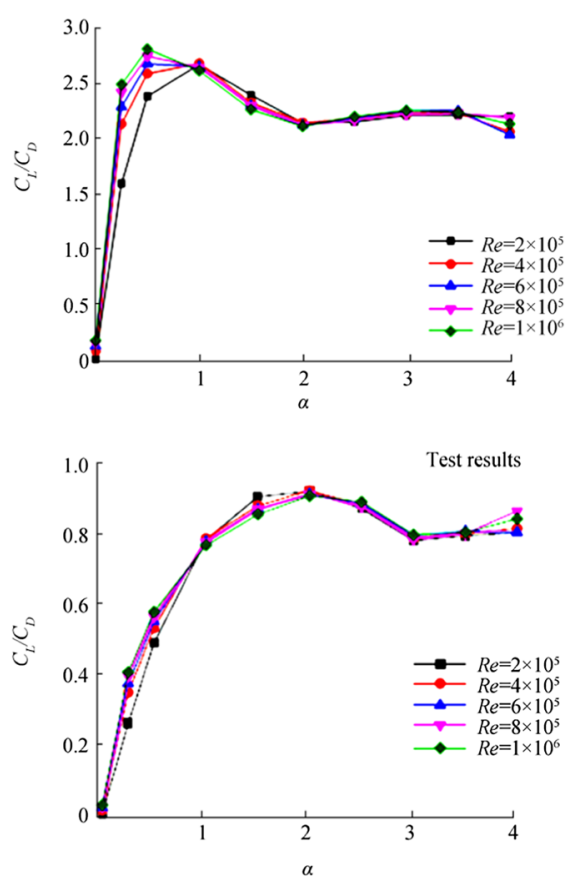

(c) $C_{L} / C_{D}$

Figure 8 Relationships among lift coefficient, drag coefficient, and lift-to-drag ratio with speed ratios under different Reynolds numbers (compared with the test results) 
increase in the length-diameter ratio, the rotational speed ratio required to reach the maximum lift-to-drag ratio became smaller and smaller, whereas the lift-to-drag ratio became larger and larger. However, larger is not better in the case. Upon observing the experimental phenomenon, we found that the vortex-induced vibration under the same rotational speed ratio became increasingly obvious with an increase in the length-diameter ratio. Furthermore, even if the rotational speed ratio is greater than 4 , the smooth progress of the experiment can still be affected, thus causing some degree of damage to the device.

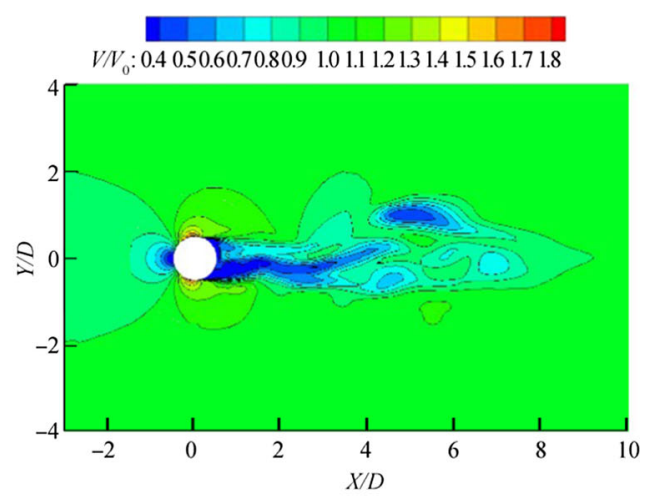

(a) $\alpha=0$

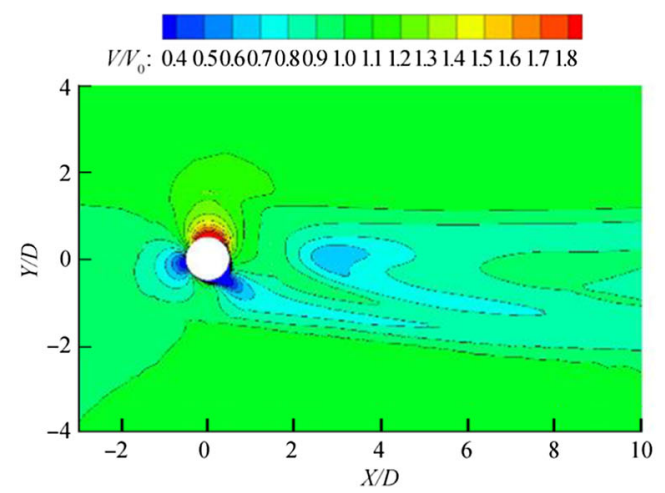

(b) $\alpha=1$

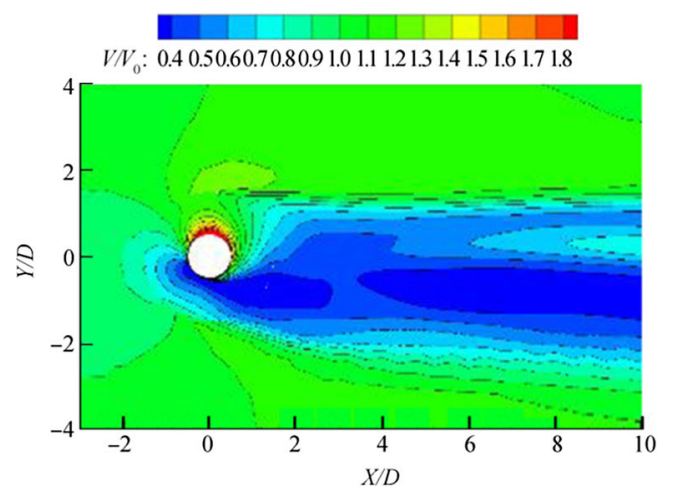

(c) $\alpha=3$

Figure 9 Flow field distribution diagram of different speed ratios under $R e=6 \times 10^{5}$
Figure 7 shows the flow field diagram of PIV measured by the test. The corresponding working condition was $R e=2.5 \times$ $10^{4}$ and $a=4$. As can be seen, the velocity in measurement area $\mathrm{A}$ was significantly higher than that in measurement area $\mathrm{B}$, resulting in the pressure in area $\mathrm{B}$ being greater than that in area A, which was the source of lateral force. Moreover, as can be seen from the vector diagram in area $\mathrm{B}$, an obvious vortex emerged in area B. This was the inevitable result of the rotation direction being opposite to the direction of incoming flow.

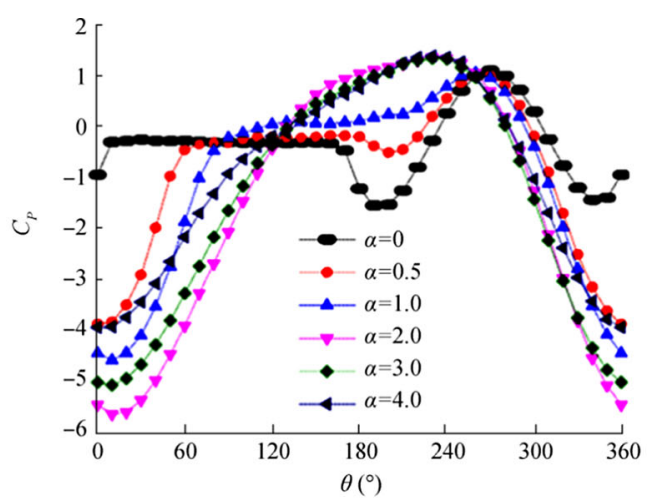

(a) $Z / D=0$

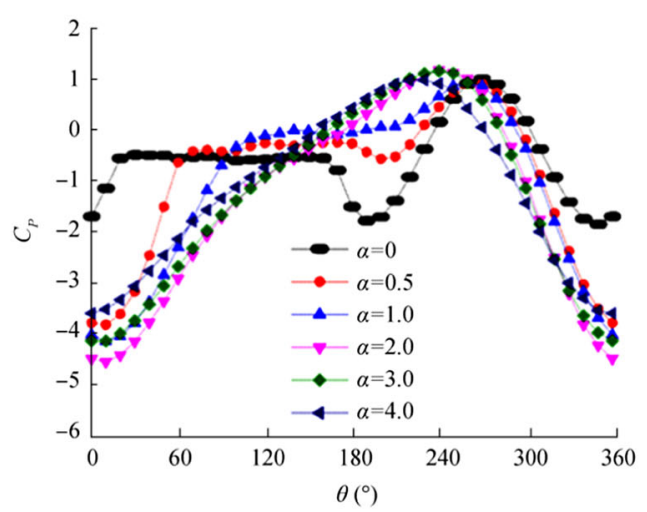

(b) $Z / D=-1.25$

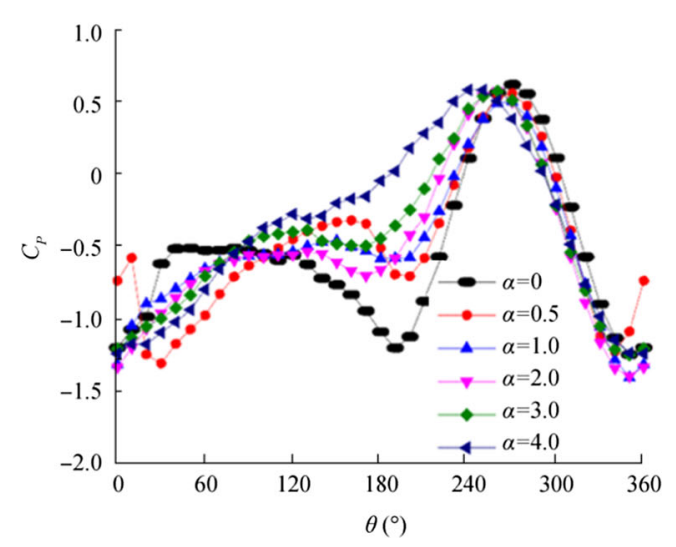

(c) $Z / D=-2.5$

Figure 10 Pressure coefficient distribution map at different cross-section positions 


\section{Analysis of Numerical Simulation Results}

\subsection{Effect of Speed Ratio on Hydrodynamic Performance}

Figure 8 presents the changes in the lift coefficient, drag coefficient, and the lift-to-drag ratio against the speed ratio, with an aspect ratio of $\beta=5$. As can be seen, the lift coefficient showed a consistent pattern as that of the changes in speed ratio for the 3D infinite-length cylinder. However, the speed ratio corresponding to the largest lift coefficient exhibited a slight deviation, and the lift coefficient was highest when the speed ratio was close to 3 . With an aspect ratio of $\beta=5$, the lift

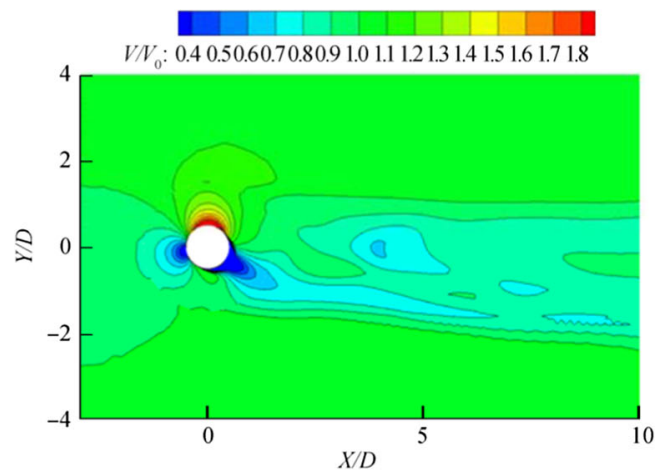

(a) $R e=2 \times 10^{5}$

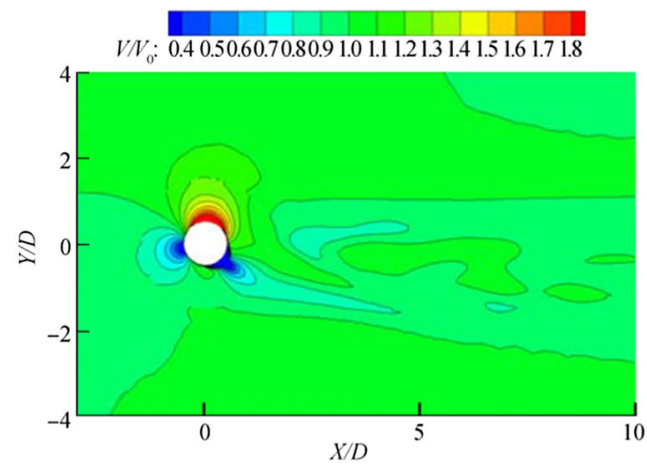

(b) $\operatorname{Re}=6 \times 10^{5}$

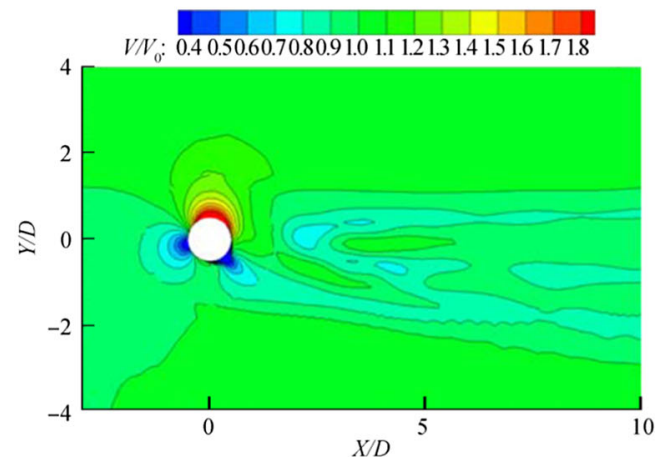

(c) $\operatorname{Re}=1 \times 10^{6}$

Figure 11 Flow patterns of the different $R e$ flow fields for a speed ratio $\alpha$ $=1$ coefficient was highest when the speed ratio $\alpha$ was approximately 2. However, as indicated in the graph, the drag coefficient ran in the opposite direction of the speed ratio. As the speed ratio increased, the drag coefficient initially increased before it started to decrease. The lift-to-drag ratio of the rotating cylinder initially increased with a rise in the speed ratio and subsequently exhibited a slight decrease before it plateaued. Results indicated that it reached its highest value when the speed ratio was in the range of 0.5-1. Compared with the experimental data, the trends of the simulation were similar; however, the specific values differed.

Figure 9 shows the flow field distribution diagram for a rotating cylinder, with $L / D=5$ calculated under $\operatorname{Re}=6 \times$ $10^{5}$, and after having attained the relative stability at different speed ratios (cross-sectional location at $Z / D=-1.25$, where $Z$

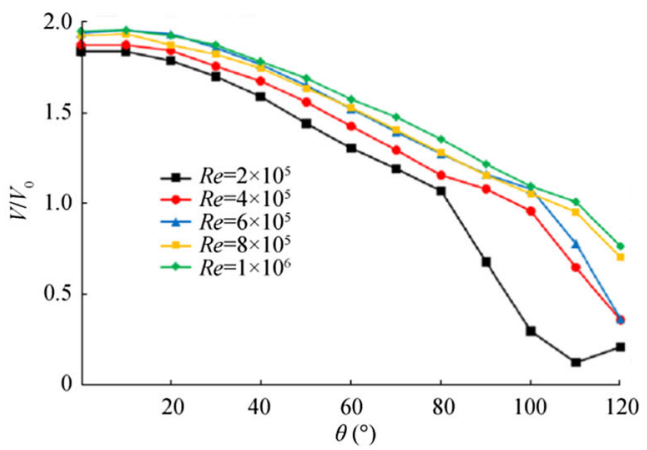

(a) $0^{\circ}-120^{\circ}$

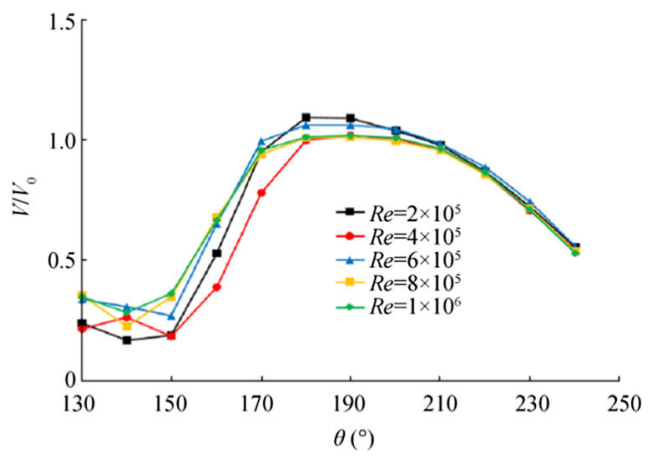

(b) $130^{\circ}-240^{\circ}$

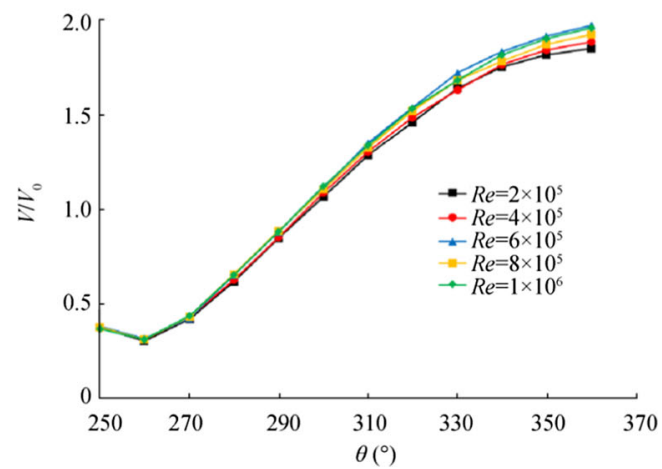

(c) $250^{\circ}-360$

Figure 12 Dimensionless velocity distribution under different values of Re 
represents the coordinates in the direction of the length). As the speed ratio increased, the flow separation point moved around the bottom right, and once the speed ratio exceeded 2 , it moved across the bottom of the cylinder toward the bottom left. This explains why the drag coefficient initially increased before it decreased. As velocity and pressure are negatively correlated with each other, a measurement was obtained at $0.505 D$ of the radius, with a pressure coefficient taken at every interval of $10^{\circ}$, to generate Figure $10(\mathrm{~b})$. As the $3 \mathrm{D}$ finite-length is different from 3D infinite-length, the difference in the flow field (pressure field) at different crosssectional locations was relatively large. Therefore, in order to generate Figure 10(a) and (c), the results were compiled at $Z / D=0$ and -2.5 , respectively. From the diagrams, for a speed ratio in the range of $0-2$, and at the same location, the pressure coefficient changed in one direction (either increasing or decreasing throughout). Once the speed ratio exceeded 2, it changed directions again. The drag coefficient experienced by the cylinder was the difference in the pressure coefficient integral between the ranges of $0^{\circ}-180^{\circ}$ and $180^{\circ}-360^{\circ}$. By scrutinizing these three diagrams, the drag coefficients were the smallest and when the values of speed ratio $\alpha$ were 0 and 2 , respectively.

\subsection{Effect of the Reynolds Number on Hydrodynamic Performance}

As shown in Figure 9, the influence of the Reynolds number was significantly less than that of the speed ratio. When the speed ratio was in the range of $0-2$, the increase in $R e$ resulted in an increase of the lift coefficient; however, the change was relatively small. When the drag coefficient was in the range of $0-0.5$, the lift coefficient decreased with increasing $R e$. Therefore, within this range, the lift-to-drag ratio increased slightly with an increasing $R e$. Meanwhile, when the speed ratio exceeded $1, R e$ had a significantly small effect on the lift and drag coefficients. Figure 11 shows the flow field distribution diagram at the location of $Z / D=-1.25$ at different $R e$ values and for a speed ratio $\alpha=1$. As can be seen, as the $R e$ increased, the value of the dimensionless velocity at the upper part of the cylinder gradually increased, although its change became less evident when $R e$ exceeded a certain value. Additionally, as $R e$ increased, the low-velocity zone (bottom right of the diagrams) decreased, and the location of the separation point appeared to move in a clockwise direction. To further investigate the effect of $R e$ on the flow field, the points were considered at $0.6 \mathrm{D}$ of the radius. The results are presented in Figure 12. As can be seen, the effect of $R e$ on the flow field manifested mainly within the range of $350^{\circ}-150^{\circ}$. In this range, the value of the dimensionless velocity increased with increasing $R e$. In comparison, such an effect was significantly small at other locations.

\subsection{Effect of Aspect Ratio on Hydrodynamic Performance}

Figure 13 shows changes in the lift coefficient, drag coefficient, and lift-to-drag ratio with speed ratio under different
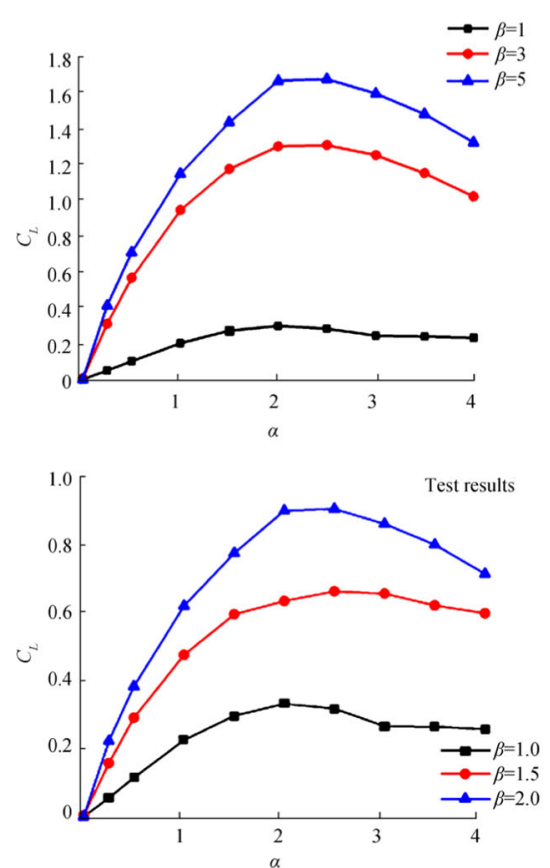

(a) $C_{L}$
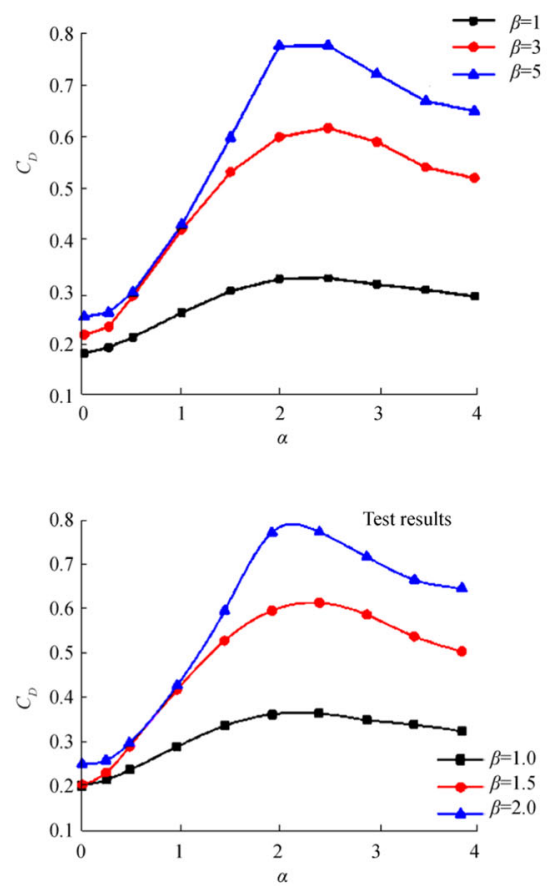

(b) $C_{D}$
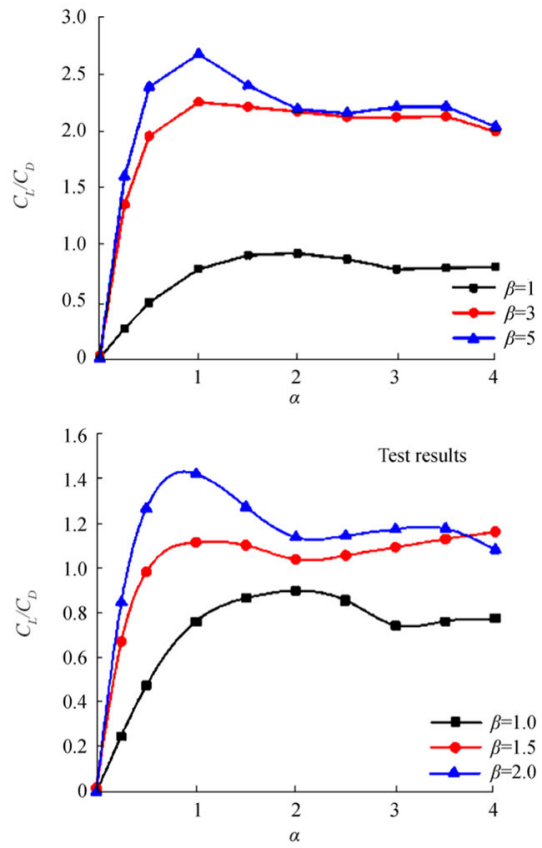

(c) $C_{L} / C_{D}$

Figure 13 Lifting coefficient, drag coefficient, and lift-to-drag ratio with different aspect ratios (compared with the test results) 


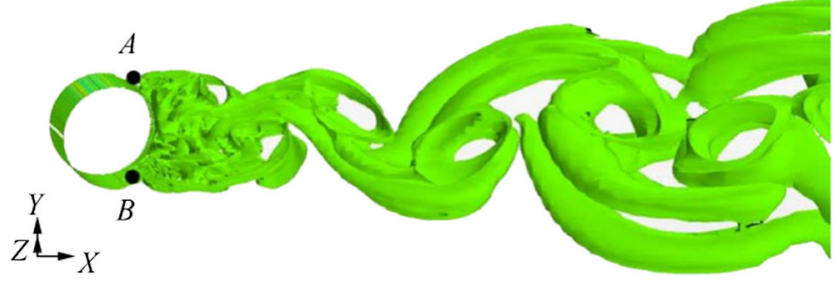

Figure 14 Vortical structures revealed by the isosurface of the $Q$ criterion $(Q=6)$

aspect ratios. As indicated in the figure, as the aspect ratio increased, the lift and drag coefficients of the rotating cylinder increased simultaneously, both reaching their highest value at a speed ratio of $\alpha=2$. The lift-to-drag ratio also increased with increasing aspect ratio, reaching its highest value at a speed ratio of $\alpha=1$. However, for the cases in which the aspect ratio was $\beta=3.5$, the lift-to-drag ratios were relatively close when $\alpha>2$. Therefore, considering both efficiency and the effects, an aspect ratio of $\beta=5$ is considered as the better alternative. The results of the two methods were similar even though there were some differences in the working conditions in the experiment.

Figure 14 shows the vorticity isosurface of the flow around the cylinder at $R e=10^{6}$. As can be clearly seen, when the fluid flowed through the cylinder, it was separated on the upper and lower surfaces, and the tail of the flow around the cylinder obviously changed from laminar flow to a turbulent state. At this time, the boundary layer on the surface of the cylinder also changed: starting from the leading edge of the cylinder, it transitioned to a turbulent mixed boundary layer through the middle transition zone after passing through the laminar flow in the front section, after which the boundary layer separation occurred. The upper and lower surfaces had two different separation points (A and B). The velocity gradient here was considerably large. Furthermore, there was a free shear layer, which was extremely unstable and had a concentrated vorticity wherein many vortices of different scales were formed. Once these vortices were generated, they were carried away by the incoming flow on the left and escaped downstream to form the wake vortex region, as shown in Figure 14.

\section{Conclusions}

Numerical simulations and a model test were performed in this study to investigate the parameters affecting the hydrodynamic performance of rotating cylinders. CFD was used to analyze the effects of the speed ratio, the Reynolds number, and the aspect ratio on the hydrodynamic performance of a 3D finite-length cylinder. We reached the following conclusions:

1 In relation to the 3D finite-length, as the speed ratio increases, the lift coefficient of a rotating cylinder will show an initial increase before it drops. The lift coefficient reaches its peak when the speed ratio $\alpha$ is 2 .

2 As the speed ratio increases, the drag coefficient of the 3D finite-length first increases before it decreases.

3 Within the range of the Re studied here, the effect of the Re on the hydrodynamic performance of the rotating cylinder is small.

4 Both the lift and drag coefficients increase as the aspect ratio increases. However, when the rotating speed reaches a certain value $(\alpha>2)$, the lift-to-drag ratio at $\beta=3$ does not differ significantly from that at $\beta=5$.

5 By comparing the results of the numerical simulation with the experimental results, we have proven that the variation trends of the drag and lift coefficients with each parameter are similar, thus verifying the reliability of the numerical simulation.

Funding Supported by the National Natural Science Foundation of China under Grant Nos. 51709060 and 51609030

Open Access This article is licensed under a Creative Commons Attribution 4.0 International License, which permits use, sharing, adaptation, distribution and reproduction in any medium or format, as long as you give appropriate credit to the original author(s) and the source, provide a link to the Creative Commons licence, and indicate if changes were made. The images or other third party material in this article are included in the article's Creative Commons licence, unless indicated otherwise in a credit line to the material. If material is not included in the article's Creative Commons licence and your intended use is not permitted by statutory regulation or exceeds the permitted use, you will need to obtain permission directly from the copyright holder. To view a copy of this licence, visit http://creativecommons.org/licenses/by/4.0/.

\section{References}

Chen BB, Luo ZH, Yuan ZY, Jiang XL, Chen B (2018) Numerical simulation of magnus effect on rotating projectile. Prog Aeronautical Eng 9(2):184-190 (in Chinese)

Cheng M, Ling GP, Zhuang YG (1990) Numerical simulation of uniform flow separated by rotating cylinder. Hydrodyn Res Progress 5(1): 65-73 (in Chinese)

Du X (2016) Magnus design and control research of anti-rolling device based on Magnus effect. PhD thesis, Harbin Engineering University. (in Chinese)

Fleming PD, Probert SD (1984) The evolution of wind-turbines: an historical review. Appl Energy 18(3):163-177. https://doi.org/10.1016/ 0306-2619(84)90007-2

Guo QS (1988) Variational principle and generalized variational principle of hybrid proposition for incompressible flow in a rotating cylindrical cascade. J Gansu Univ Technol 14(2):44-50 (in Chinese)

Islam SU, Zhou CY, Shah A, Xie P (2012) Numerical simulation of flow past rectangular cylinders with different aspect ratios using the incompressible lattice Boltzmann method. J Mech Sci Technol 26(4): 1027-1041. https://doi.org/10.1007/s12206-012-0328-4

Karabelas SJ (2010) Large eddy simulation of high-Reynolds number flow past a rotating cylinder. Int J Heat Fluid Flow 31(4):518-527. https://doi.org/10.1016/j.ijheatfluidflow.2010.02.010 
Liang L, Zhao P, Zhang S (2016) Research on hydrodynamic characteristics of Magnus rotor wing at medium/low speed. 2016 IEEE International Conference on Mechatronics and Automation. IEEE, Harbin, 2413-2418.

Liu XY, Zhuang LX (1994) Numerical study of viscous flow around a rotating cylinder in uniform incoming flow. Acta Mech Sinica 26(2):233-238 (in Chinese)

Ommani B, Kristiansen T, Faltinsen OM (2016) Simplified CFD modeling for bilge keel force and hull pressure distribution on a rotating cylinder. Appl Ocean Res 58:253-265. https://doi.org/10.1016/j. apor.2016.04.010

Sedaghat A, Ali Badri M, Saghafian M, Samani I (2014) An innovative Treadmill-Magnus wind propulsion system for naval ships. Recent Pat Eng 8(2):95-99. https://doi.org/10.2174/ 1872212108666140530231724

Siddiqui AA (2016) Accelerated micropolar fluid-flow past an uniformly rotating circular cylinder. AIP Adv 6(10):105101. https://doi.org/10. $1063 / 1.4964493$

Tu CX, Wang HL, Lin JZ (2008) Experimental research on the flow characteristics and vortex shedding in the flow around a circular cylinder. J China Jiliang Univ 19(2):98-102 (in Chinese)

van Rees WM, Novati G, Koumoutsakos P (2015) Self-propulsion of a counter-rotating cylinder pair in a viscous fluid. Phys Fluids 27(6): 063102. https://doi.org/10.1063/1.4922314

Wong KWL, Zhao J, Jacono DL, Thompson MC (2017) Experimental investigation of flow-induced vibration of a rotating circular cylinder. J Fluid Mech 829:486-511. https://doi.org/10.1017/jfm. 2017.540

Wu F, Wang XD, Chang SJ, Liu SJ (2018) Numerical simulation of magnus effect of rotating tail projectile. Acta Ballistics Sinica 30(1):12-18 (in Chinese)

Xu HZ, Liu YH (1988) Hydrodynamic study of rotating column rudder. Chinese Shipbuilding 1(2):31-39 (in Chinese)

Xu HZ, Sun YB (1992) Application of rotating rudder on real ship. Chinese Shipbuilding 2(3):26-34 (in Chinese)

Xu HB, Shen YW, Jun Z (1995) Study on Taylor vorticity between infinitely long eccentric rotating cylinders. J Northwest Polytech Univ 13(2):168-173 (in Chinese)

Yuan ML, 2016. Numerical simulation of Magnus effect wind turbine aerodynamic performance and exploration of airfoil optimization. $\mathrm{PhD}$ thesis, Huazhong University of Science and Technology. (in Chinese)

Zhang RJ, Xu HZ (1991) Model test analysis of rotating column rudder series. Mar Eng 2(4):32-38 (in Chinese)

Zheng XR, Xu J (1993) Wave rotor using wave power. Sol Energy 1(2): 24 (in Chinese)

Zheng Z, Lei J, Wu X (2017) Numerical simulation of the negative Magnus effect of a two-dimensional spinning circular cylinder. Flow Turbul Combust 98(1):109-130. https://doi.org/10.1007/ s10494-016-9747-0 